\title{
Serum Gp96 is a chaperone of complement-C3 during graft-versus-host disease
}

Antoine Seignez, ${ }^{1,2,3}$ Anne-Laure Joly, ${ }^{1,2}$ Killian Chaumonnot, ${ }^{1,2}$ Adonis Hazoumé, ${ }^{1,2}$ Michel Sanka, ${ }^{1,2}$ Guillaume Marcion, ${ }^{1,2}$ Christophe Boudesco, ${ }^{1,2}$ Arlette Hammann, ${ }^{1,2}$ Renaud Seigneuric, ${ }^{1,2}$ Gaetan Jégo, ${ }^{1,2}$ Patrick Ducoroy, ${ }^{4}$ Patrice Delarue, ${ }^{5}$ Patrick Senet, ${ }^{5}$ Cristina Castilla-Llorente, ${ }^{6}$ Eric Solary, ${ }^{6,7}$ Marie-Agnès Durey, ${ }^{8}$ Marie-Thérèse Rubio, ${ }^{9,10}$ Olivier Hermine, ${ }^{11,12}$ Evelyne Kohli, ${ }^{1,2,3}$ and Carmen Garrido

'INSERM UMR 866, Equipe labellisée, Ligue Nationale contre le Cancer, and Laboratoire d'Excellence LipSTIC, Dijon, France. ${ }^{2}$ Université de Bourgogne Franche-Comté, LNC UMR 866, Dijon, France. ${ }^{3} \mathrm{CHU}$, Dijon, France. ${ }^{4}$ Proteomic platform CLIPP, Université de Bourgogne Franche-Comté, Dijon, France. ${ }^{5}$ UMR 6303 CNRS Institut Carnot, Université de Bourgogne Franche-Comté, Dijon, France. 'Institute Gustave Roussy, Université Paris-Sud 11, Villejuif, France. ' INSERM UMR1009, Institute Gustave Roussy, Villejuif, France. ${ }^{8}$ Immunology Department, Hôpital Européen Georges Pompidou, Assistance Publique-Hôpitaux de Paris, Paris, France; Université Paris Descartes, Paris, France. ${ }^{9}$ Service d'Hématologie et Thérapie Cellulaire, Hôpital Saint-Antoine, Assistance Publique-Hôpitaux de Paris, Paris, France. ${ }^{10}$ INSERM UMR 938, Université Pierre et Marie Curie, Paris, France. "Institut Imagine, UMR 8147, Université Paris Descartes, Sorbonne Paris-Cité; Hôpital Necker, Assistance publique-Hôpitaux de Paris, Paris, France. '2Laboratoire d'Excellence des Globules Rouges (GR-ex), Paris, France. ${ }^{13}$ Anticancer Centre Georges-François Leclerc, Dijon, France.

Better identification of severe acute graft-versus-host disease (GvHD) may improve the outcome of this life-threatening complication of allogeneic hematopoietic stem cell transplantation. GvHD induces tissue damage and the release of damage-associated molecular pattern (DAMP) molecules. Here, we analyzed GvHD patients $(n=39)$ to show that serum heat shock protein glycoprotein 96 (Gp96) could be such a DAMP molecule. We demonstrate that serum Gp96 increases in gastrointestinal GvHD patients and its level correlates with disease severity. An increase in Gp96 serum level was also observed in a mouse model of acute GvHD. This model was used to identify complement C3 as a main partner of Cp96 in the serum. Our biolayer interferometry, yeast twohybrid and in silico modeling data allowed us to determine that Gp96 binds to a complement C3 fragment encompassing amino acids 749-954, a functional complement C3 hot spot important for binding of different regulators. Accordingly, in vitro experiments with purified proteins demonstrate that Gp96 downregulates several complement C3 functions. Finally, experimental induction of CvHD in complement C3-deficient mice confirms the link between Gp96 and complement C3 in the serum and with the severity of the disease.

Authorship note: AS and ALJ are co-first authors. EK and CG are co-senior authors.

Conflict of interest: The authors have declared that no conflict of interest exists.

Submitted: September 6, 2016 Accepted: February 8, 2017 Published: March 23, 2017

Reference information: JCI Insight. 2017;2(6):e90531. https:// doi.org/10.1172/jii.insight.90531

\section{Introduction}

Endogenous noninfectious molecules, including intracellular proteins, are part of the damage-associated molecular pattern (DAMP) molecules, also called alarmins, that are released by stressed tissues and act as endogenous danger signals to promote inflammatory and immune responses. In the setting of allogeneic hematopoietic stem cell transplantation, such molecules can be released during the conditioning regimen and activate host antigen-presenting cells that, in turn, stimulate and polarize donor T cells; this can lead to acute graft-versus-host disease (GvHD), the major complication of this therapeutic approach $(1,2)$. Acute GvHD is the result of alloreactive donor T cells attacking host tissues (mainly gastrointestinal tract, skin, and liver; ref. 3). DAMP molecules released by these damaged tissues perpetuate and amplify the acute $\operatorname{GvHD}(1,4)$.

Heat shock proteins (Hsps) are conserved chaperones involved in the proper folding, assembly, and turn-over of various proteins (5). Hsps are constitutively present in various cells, and their expression increases as a cytoprotective response to environmental stress. Hsps were involved in acute GvHD 
pathogenesis, i.e., Hsp70 protein expression was associated with skin damage in an in vitro-generated GvHD reaction in human skin (6), whereas Hsp90, whose expression increases in activated T cells, was proposed as a target for selective depletion of alloreactive T cells $(7,8)$.

Some Hsps are released during cell stress and injury and behave as DAMPs (9). One of these Hsps is glucose-regulated protein Gp96 (encoded by HSP90B1), known also as GRP94 or tumor rejection antigen 1, which is a paralog of HSP90 in the endoplasmic reticulum. Gp96 intracellular level rises during stress conditions such as inflammation, hypoxia, or glucose starvation (10). Gp96 binds and hydrolyzes ATP, is the most abundant protein in the endoplasmic reticulum, and is ubiquitously expressed in all nucleated cells. Intracellular Gp96 acts as a molecular chaperone able to favor protein folding and either protect or inactivate bound proteins (11). Under certain stress conditions, Gp96 is also found outside the cells and on the membrane of some immune cells. Gp96 extracellular functions strongly suggest that, once exiting the cell, Gp96 may behave as a DAMP molecule (12). Gp96 promotes innate immune response activation by proinflammatory cytokines/chemokines $(13,14)$. In addition, extracellular Gp96 increases specific immune response by loading peptides onto MHC molecules and facilitating antigen presentation to T cells $(15,16)$.

Here, we show that Gp96 serum level increases as GvHD develops in patients receiving hematopoietic stem cell transplantation, as well as in a mouse model of acute GvHD. We demonstrate that, in the serum, Gp96 binds to and chaperones complement C3 fraction, a central functional hub that largely drives the activation, amplification, and effector generation of the complement response.

\section{Results}

Gp96 level increases in the sera of patients developing acute $G v H D$. We first quantified Gp96 by ELISA in the sera of patients with acute GvHD (gastrointestinal and/or skin GvHD; $n=39$ ). Patients' clinical characteristics are presented in Table 1. We found that Gp96 concentration in the serum significantly increased 7 days after clinical diagnosis of GvHD (GvHD onset), compared with levels observed on day 7 before the diagnosis (Figure 1A). This increase did not correlate with the type of conditioning regimen (chemotherapy and/ or total body irradiation or the response to corticotherapy). Gastrointestinal GvHD is the worst form of the disease, leading to an increase of the morbidity and mortality. Therefore, for the intestinal GvHD patients for which we had a clinical consensus regarding GvHD grade about the GvHD grade $(n=17)$, we determined Gp96 levels in grade II versus grade III-IV. Interestingly, we found that Gp96 increase concerned the severe forms of intestinal GvHD (grade III-IV) (Figure 1B). In contrast to these results, no changes in Gp96 levels were observed in patients where isolated skin GvHD was diagnosed ( $n=18$; Figure 1C). As a supplementary control, we also analyzed patients that did not develop GvHD $(n=7)$. We measured Gp96 3 weeks after hematopoietic stem cell transplantation (day 21 [D21], most often the onset of GvHD) and 1 week later (D28, corresponding to D+7 in the GvHD patients). We did not find any increase in Gp96 levels in the sera of these patients (Figure 1D). These encouraging results prompted us to study Gp96 in gastrointestinal GvHD.

Gp96 increases in the sera of mice developing acute GvHD. We next used a mouse model of acute GvHD reproducing all the signs of gastrointestinal GvHD (Figure 2A, left panel and ref. 7). Recipient BALB/c animals were irradiated before bone marrow + spleen-derived $\mathrm{T}$ cell transplantation from allogenic C57BL/6 animals (Allo group). As controls that do not develop the GvHD, we used animals transplanted with only bone marrow cells (BM group) or with bone marrow + spleen-derived T cells from syngeneic animals (Syn group). As expected, BM and Syn animals did not develop a GvHD, while the Allo group developed the disease (all animals of the Allo group died around 15 days after graft [Figure 2A, right panel]). Gp96 was assessed in the mice sera by Western blotting. The results showed that Gp96 but not HSP90, a cytosolic member of the HSP90 family, was increased at D7 in the sera from mice developing a GvHD. No increase was observed in the control Syn and BM mice that did not develop the disease (Figure 2B). We then analyzed Gp96 increase at different times after transplantation and demonstrated that Gp96 levels peaked at D7 in the Allo mice sera (Figure 2C). In addition, we found that Gp96 was also overexpressed 7 days after transplantation at the membrane of splenic $\mathrm{F} 4 / 80^{+}$cells isolated from Allo mice compared with $\mathrm{BM}$ and Syn mice (Figure 2D).

Gp96 binds to complement $C 3$ in the serum during GvHD. To identify Gp96-associated proteins in the serum during GvHD development, we immunoprecipitated Gp96 from mice's sera (Figure 3A) and identified the bound proteins after SDS-PAGE by comparing bands between Allo mice developing GvHD and control groups (mice receiving a syngeneic graft [Syn] and nongrafted mice). Three bands were repetitively 
Table 1. GvHD Patients' clinical characteristics $(n=39)$.

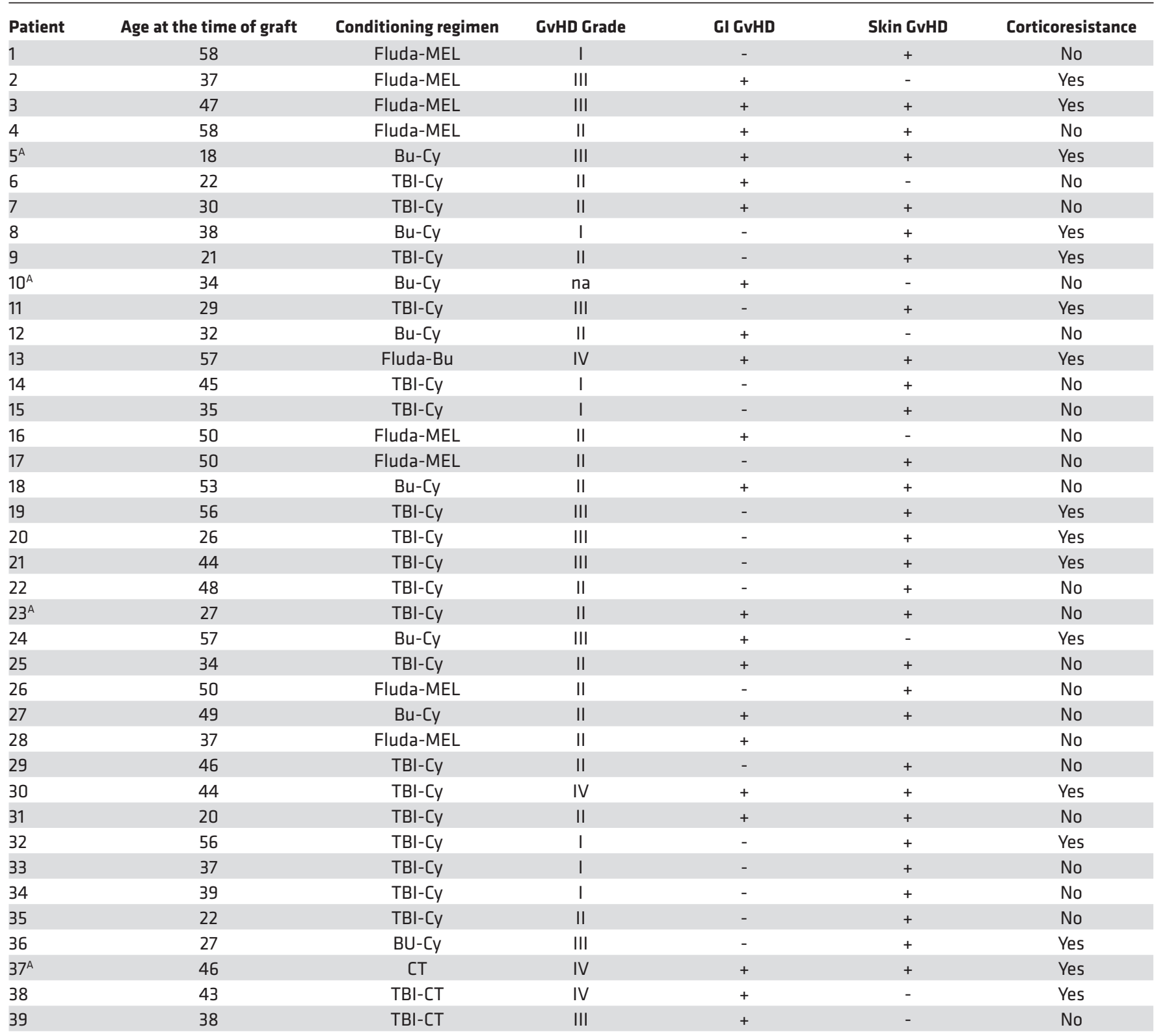

TBI, total body irradiation (12 Gy); Fluda, fludarabine; MEL, melphalan; Bu, busulfan; Cy, cyclophosphamide; CT, chemotherapy. ${ }^{A}$ Patients not included in Figure $1 \mathrm{~B}$ because, in patient 10 , the grade was not available, and in patients 5, 23, and 37, skin and GI-GvHD were diagnosed at the same time.

observed exclusively in the sera of animals developing the GvHD (Allo mice) (Figure 3B). Analysis of those bands by mass spectrometry (MS) revealed that all 3 corresponded to the complement C3 (Supplemental Figure 1; supplemental material available online with this article; https://doi.org/10.1172/jci. insight.90531DS1). The fact that complement C3's bands were hardly present in the control groups that do not develop GvHD or when using a nonrelevant antibody attests the specificity of the results obtained. The interaction of Gp96 with complement $\mathrm{C} 3$ was confirmed by interference biolayer with an OctetRED instrument. Our data indicate that the 2:1 model is the most suitable for fitting the experimental sensorgrams of both the $\mathrm{Gp} 96: \mathrm{C} 3$ and the $\mathrm{Gp} 96: \mathrm{C} 3 \mathrm{~b}$ (the primary cleavage complement $\mathrm{C} 3$ fragment) interactions $\left(\chi^{2}\right.$ and $\mathrm{R}^{2}$ values of 0.017 and $0.973 ; 0.020$ and 0.981 , respectively) and allowed us to determine an averaged $\mathrm{Kd}$ of $350 \mathrm{nM}$ for complement C3 (Figure 3C, left panel) and of $90 \mathrm{nM}$ for C3b (Figure 3C, right panel). Another chaperone, HSP60, was used here as a negative control. 
A

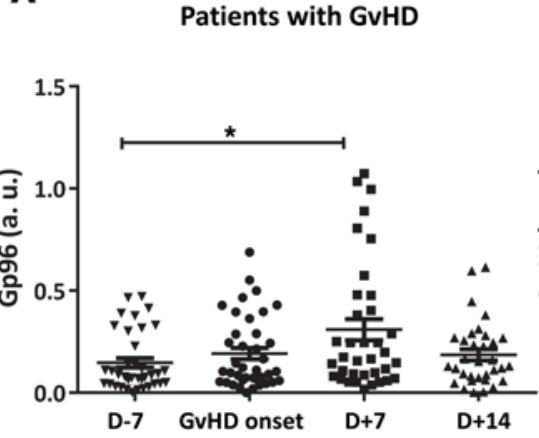

B

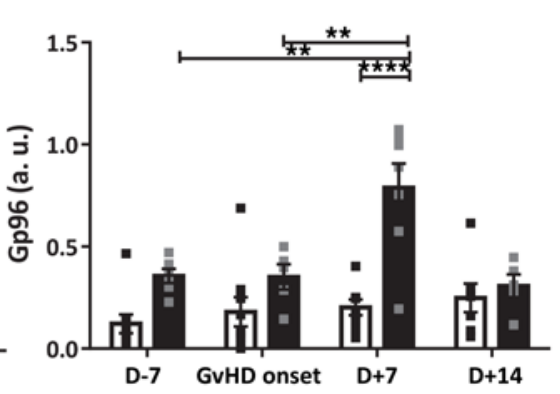

C

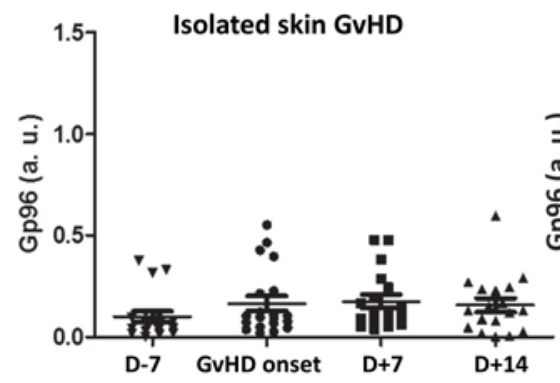

D

B $\square$ GI GvHD, grade II

GI GvHD, grade III-IV

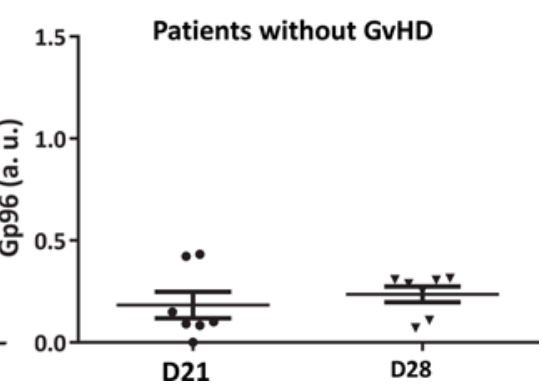

Figure 1. Gp96 expression in acute GvHD patient sera. Available sera from 39 patients were sampled 7 days before onset ( $D-7)$, the day of acute GvHD onset, and 7 days and 14 days after diagnosis $(D+7$ and $D+14$, respectively). Gp96 was quantified by ELISA. (A) All acute GVHD patients included. It is worth noting that, for some patients, data at one specific time point may be missing. Statistical analysis performed using a 1-way ANOVA test (Dunn's multiple comparisons test). ${ }^{*} P<0.05$. (B) Patients $(n=17)$ with grade II $(n=10)$ vs. III-IV intestinal (GI) GVHD $(n=7)$. Statistical analysis performed using a 2-way ANOVA test (Tukey's multiple comparisons test). ${ }^{* *} P<0.01,{ }^{* * *} P<0.0001$. (C) Patients presenting isolated skin GvHD $(n=18)$. (D) Non-GvHD patients $(n=7)$. Gp96 levels were measured 3 weeks after hematopoietic stem cell transplantation (median: D21, most often the onset of CvHD) and 1 week later (median: D28).

Full-length Gp96 binds to the 749-954 amino acid fragment of complement C3. Using a yeast two-hybrid system, we first sought to provide additional evidence for the interaction of Gp96 with complement C3. Blue diploid transformants could be detected on a high stringency minimal medium, indicating a direct interaction between Gp96 and complement C3.

To map the domains of Gp96 and complement C3 involved, we used different C3 fragments and Gp96 deletion mutants. Figure 4A represents the C3 full-length and fragments tested. We found that Gp96 interacted with the $\alpha$-chain of complement C3. More precisely, a region between amino acids 749 and 954 was the minimal fragment required for this interaction (Figure $4 \mathrm{~B}$ ). It is worth noting that this minimal fragment is common to C3, its opsonic fragment $\mathrm{C} 3 \mathrm{~b}$, and the cleavage fragment of $\mathrm{C} 3 \mathrm{~b}$ known as $\mathrm{iC} 3 \mathrm{~b}$ (Figure $4 \mathrm{~A}$ and Supplemental Figure 2). Next, we cloned either full-length Gp96 or deletion mutants lacking either the ATP-binding or the peptide-binding domain with and without the calcium-binding site that regulates the chaperone activity (Figure 4C). Interestingly, none of these 3 deletion mutants interacted with complement C3, suggesting that the entire structure of the chaperone is required (Figure 4D). Nevertheless, our in silico modeling studies implicate the N-terminal region of Gp96 in this interaction. Indeed, as shown in Figure 5, the modeling shows that there exist plausible models of full-length C3-Gp96 complexes with strong interactions between the complement C3 749-954 amino acid fragment and the N-terminus region of Gp96.

Gp96 inhibits factor I/factor $H$-mediated cleavage of C3b. As the complement C3 region involved in the interaction with Gp96 is needed for binding to factor $\mathrm{H}$, which is an important regulator of complement activity by promoting the cleavage of $\mathrm{C} 3 \mathrm{~b}$ to $\mathrm{iC} 3 \mathrm{~b}$ by factor I, we explored whether $\mathrm{Gp} 96$ could protect C3 from this enzymatic proteolysis. Purified human C3b was incubated with human factors $\mathrm{I}$ and $\mathrm{H}$ in the presence of serum with or without human recombinant Gp96 (LPS-free). Specific chains of C3b and iC $3 b$ were analyzed by SDS-PAGE. C3b cleavage after 10 minutes and 30 minutes of incubation is shown in Figure 6A. We found that, in the presence of $\mathrm{Gp} 96$, the $115 \mathrm{kDa} \alpha^{\prime}$ chain of $\mathrm{C} 3 \mathrm{~b}$ was more abundant, whereas the amount of the cleaved forms $\alpha 1-\mathrm{dg}$-chain $(67 \mathrm{kDa}, \mathrm{iC} 3 \mathrm{~b})$ and the $\alpha 2$-chain $(43 \mathrm{kDa}$, common to $\mathrm{iC} 3 \mathrm{~b}$ and $\mathrm{C} 3 \mathrm{c}$ ) were reduced.

Gp96 inhibits opsonophagocytosis of E. coli by hampering C3 tagging of bacteria's membranes. We incubated AF488-conjugated E. coli particles with complement C3 from serum of healthy donors in the presence or absence of human recombinant Gp96 and subsequently incubated the bacteria with human primary macrophages. As shown in Figure 6B, phagocytosis was inhibited when the particles were opsonized in the presence of Gp96. By immunoblot (Figure 6C), we showed that, when opsonization was performed in the presence of Gp96, there was a decrease in the amount of C3 deposited on bacteria, but not of $\mathrm{C} 4$, used here as a control. This suggests that Gp96 inhibits C3b deposition at the surface of bacteria rather than the phagocytosis of opsonized bacteria. This result was confirmed by quantification of $\mathrm{C} 3 \mathrm{~b}$ 
A
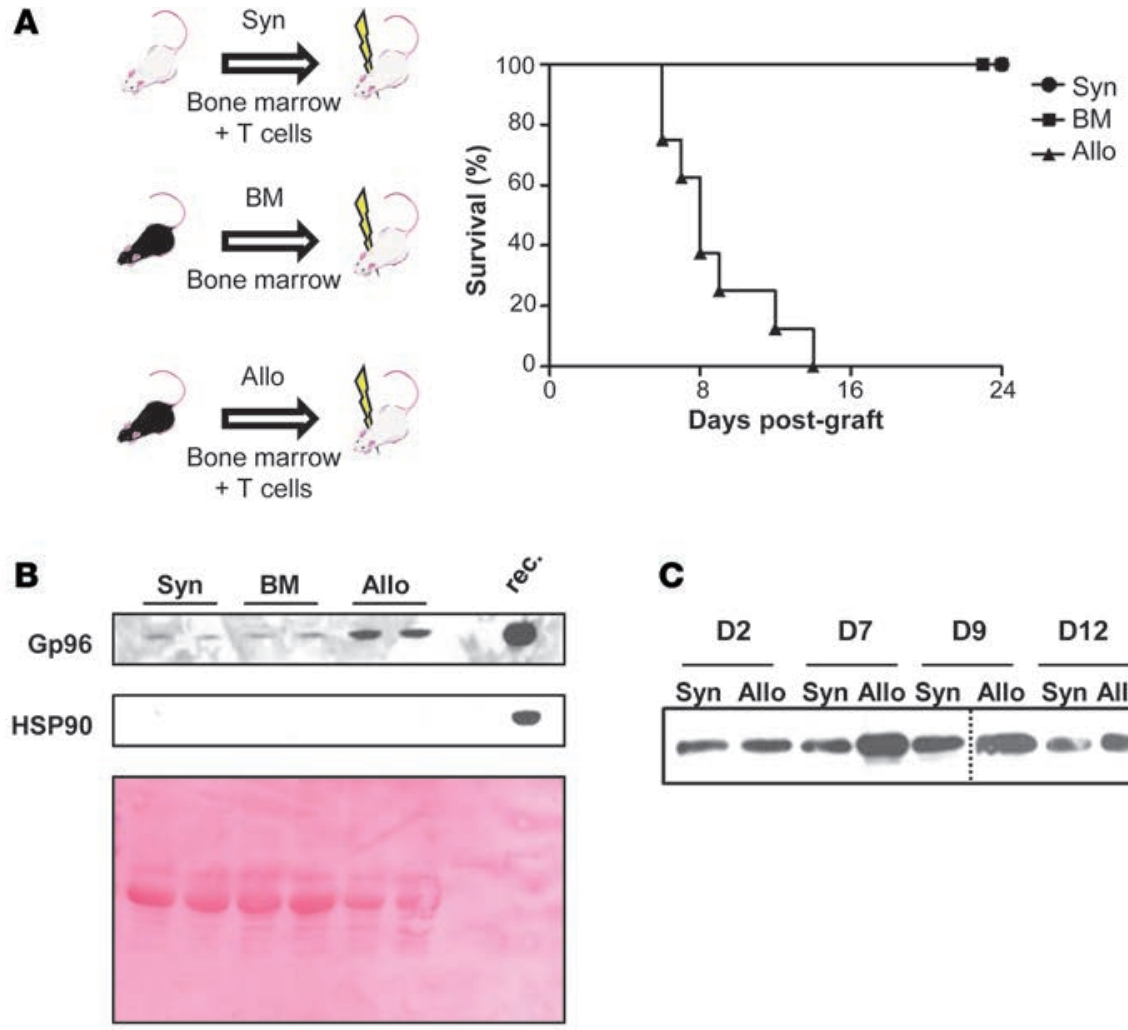

C

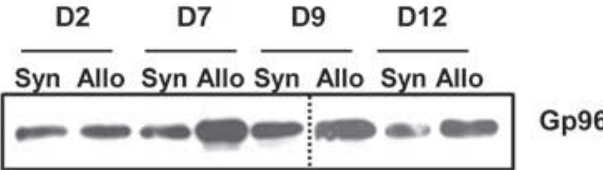

D
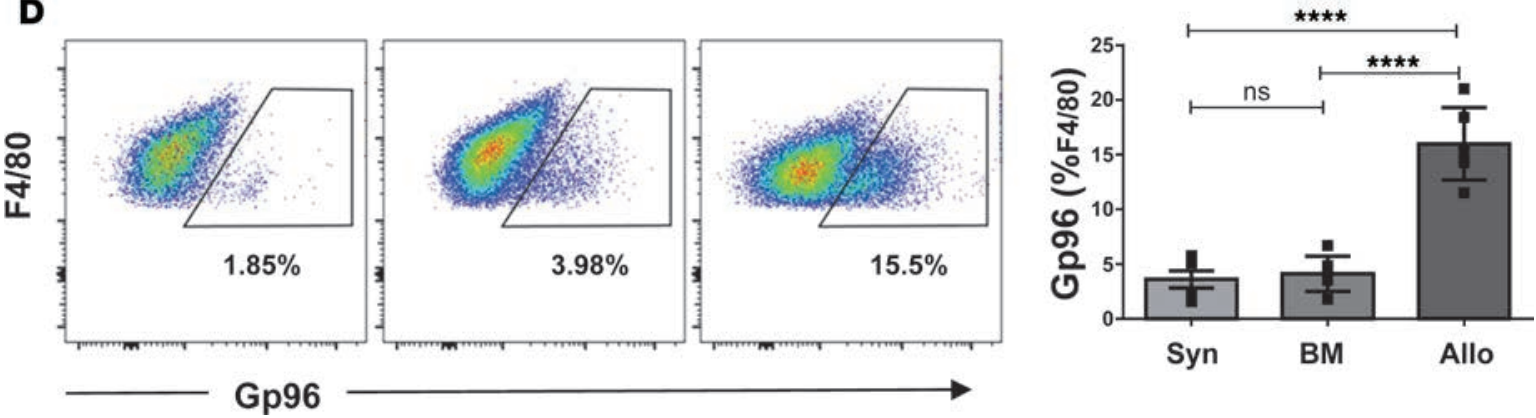

Figure 2. Gp96 expression in the murine GvHD model. (A) Left panel: mouse model of acute GvHD. Syn: syngeneic graft (no GvHD developed). BM: allogeneic graft without splenocytes (no GvHD). Allo: allogeneic graft with splenocytes (acute GvHD). Right panel: survival of the mice belonging to the different groups. (B) Western blot analysis of Gp96 and HSP9O in sera at day 7 after transplantation. Recombinant proteins were run as a control. (C) Western blot analysis of Gp96 expression at the indicated days after transplantation in sera of mice developing GvHD (Allo) or not (Syn). The lanes were run on the same gel but were noncontiguous. (D) Proportion of splenic macrophages (according to F4/80 staining) expressing Gp96 at the surface was determined by FACS analysis 7 days after hematopoietic stem cell transplantation. Statistical analysis performed using a 1-way ANOVA test. ${ }^{* * * *} P<0.0001$. A representative experiment is shown $(n=3)$.

on bacteria by microscopy after C3 A568 staining (Figure 6D). Further confirming this result, we did not observe any inhibition of phagocytosis when Gp96 was added 30 minutes after incubation of the bioparticles with complement C3 (Supplemental Figure 3).

Gp96 inhibits the alternative and the classical pathway of complement C3 activation in human serum. Initiation of the complement response by any activation route, either the classical or the alternative, leads to the formation of protein complexes called C3 convertases at the surface of the recognized cell. These convertases bind and activate $\mathrm{C} 3$ to generate the opsonic fragment $\mathrm{C} 3 \mathrm{~b}$. Increasing $\mathrm{C} 3 \mathrm{~b}$ deposition leads to the formation of C5 convertases (17). The C5 convertase of the classical pathway, as well as the C 3 and C5 convertases of the alternative pathways, contain at least $1 \mathrm{C} 3 \mathrm{~b}$ molecule that can bind Gp96. Therefore, we determined whether Gp96 could block the membrane attack complex formation by the alternative or classical convertases using complement hemolytic assays. Our results with different healthy patients' sera 
A
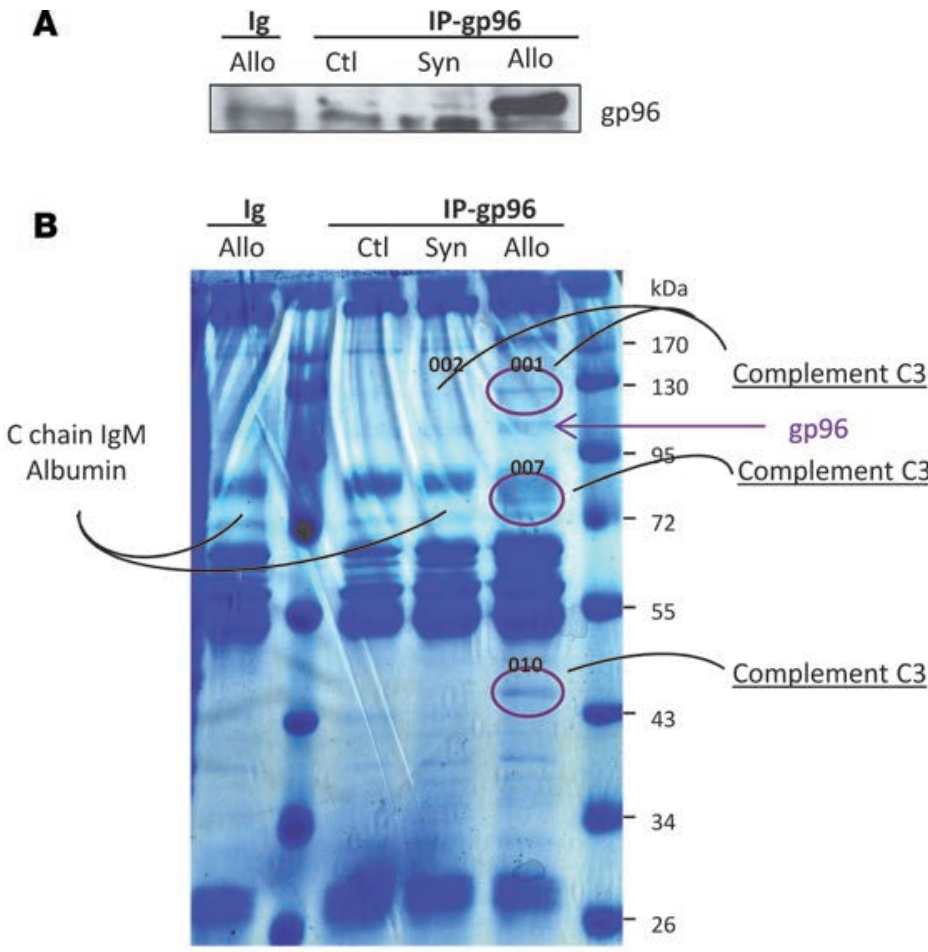

Figure 3. C3 associates with Gp96 in mouse sera during GvHD. (A) Western blot analysis of Gp96. (B) Coomassie blue coloration of proteins coimmunoprecipitated with Gp96 (bottom panel) in mice developing GvHD (Allo) or not (Syn) 7 days after hematopoietic stem cell transplantation. Ctl, mice with no transplantation. The proteins indicated were determined by mass spectrometry. One representative experiment is shown ( $n$ =2). (C) Protein-protein interaction data between Gp96 or HSP60 immobilized onto the biosensor and C3 (left panel) or C3b (right panel) as analytes.

\section{C}

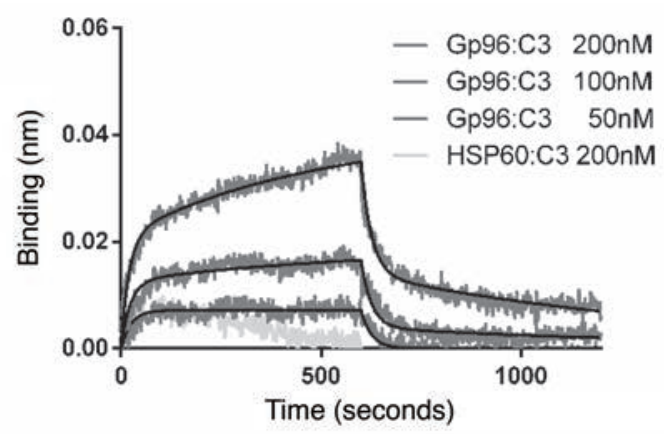

Gp96:C3b and HSP60:C3b

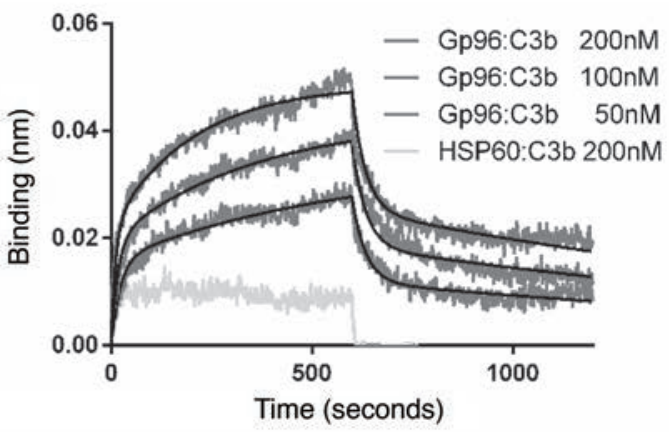

demonstrate that Gp96 inhibits hemolytic activity in serum activated by both the classical and the alternative pathways (Figure 6, E and F).

Gp96 serum level correlates with that of complement C3 and with GvHD severity in mice. To confirm in vivo that Gp96 may be a chaperone of complement C3 associated with GvHD severity, we used complement C3 KO animals (Gp96 KO animals are not viable, ref. 28). GvHD was reported to be less severe when recipient mice were knocking out for complement $\mathrm{C} 3$. We first used $\mathrm{C} 3^{-1-} \mathrm{C} 57 \mathrm{BL} / 6$ mice as recipients and $\mathrm{WT} \mathrm{FvB} / \mathrm{N}$ mice as donors. As previously reported, recipient $\mathrm{C}^{-1-} \mathrm{C} 57 \mathrm{BL} / 6$ mice developed a less severe GvHD and demonstrated a longer survival when compared with WT C57BL/6 recipients (ref. 18 and data not shown). As expected, C3 was not detected in the sera from $\mathrm{C}^{-1-}$ recipients (Figure 7A), which, interestingly, correlated with a much lower level of Gp96 compared with WT recipient Allo mice (Figure 7A). Next, we used $\mathrm{C}^{-/-} \mathrm{C} 57 \mathrm{BL} / 6$ mice as donors and WT BALB/c as recipients. Seven days after hematopoietic stem cell transplantation, Gp96 and C3 were both detected in the serum of recipient animals (Figure 7B, left panel) and the 2 proteins were confirmed to interact (Figure 7B, right panel). Interestingly, we detected more of both $\mathrm{C} 3$ and $\mathrm{Gp} 96$ in the serum of recipient Allo mice grafted with $\mathrm{C}^{3^{--}} \mathrm{BM}$ cells + splenocytes than in the serum of those grafted with WT cells. Allo mice grafted with $\mathrm{C}^{-/-} \mathrm{BM}$ cells + splenocytes developed a more severe and rapidly fatal GvHD than those grafted with WT cells (Figure 7C and data not shown for clinical scores). Accordingly, more activated $\mathrm{T}$ cells $\left(\mathrm{CD} 3^{+} \mathrm{CD} 69^{+}\right)$were detected in the spleen of Allo mice grafted with $\mathrm{C}^{-/-}$compared with WT cells (Figure 7D). It is worth noting that, in these 
A

25
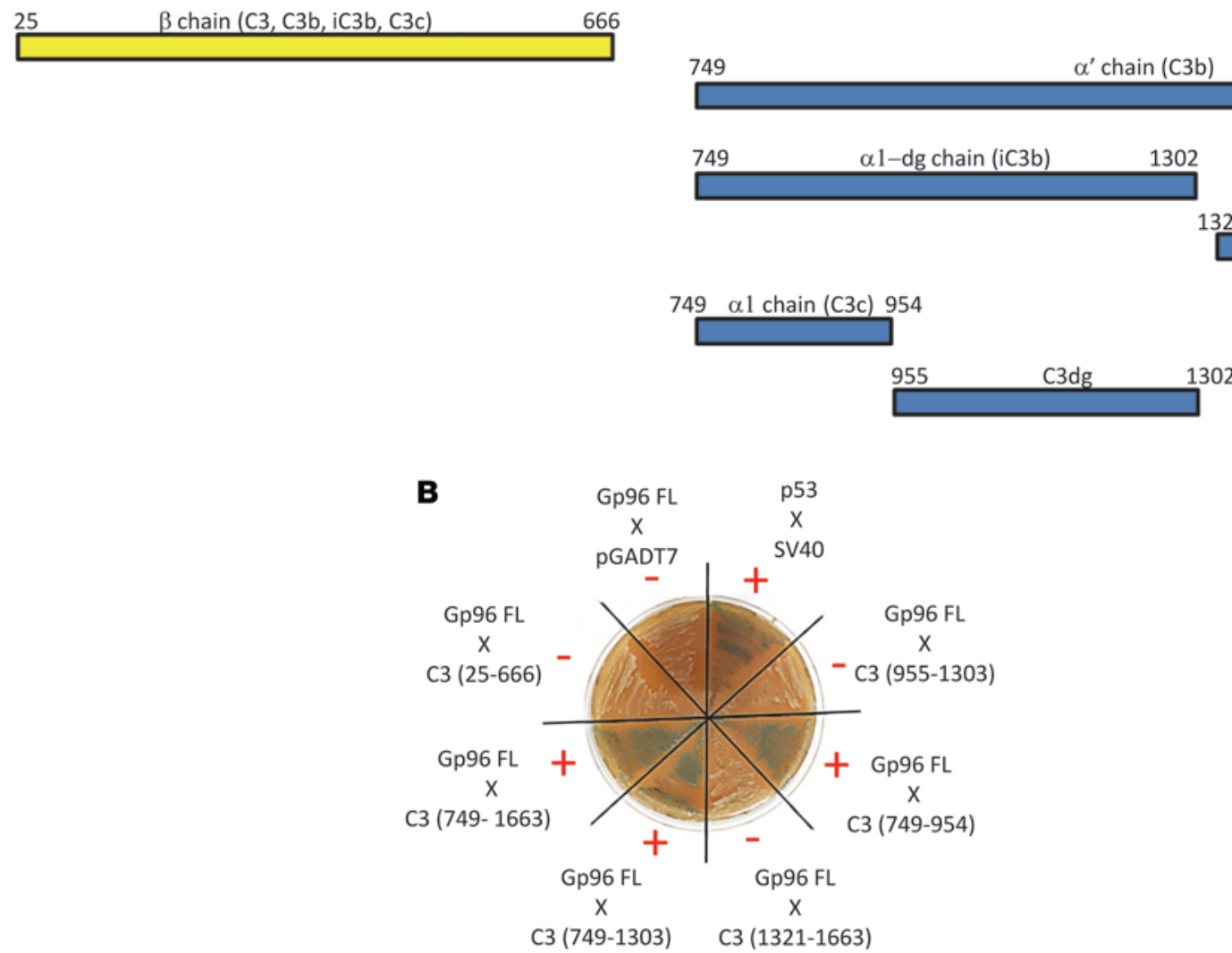

\section{C}

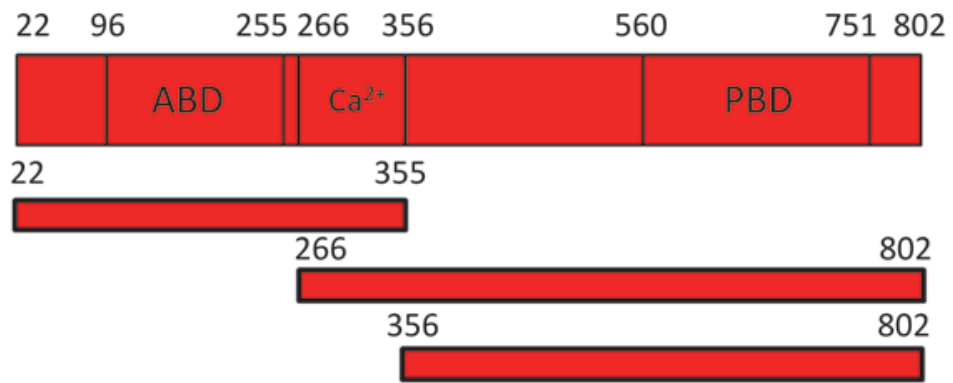

D

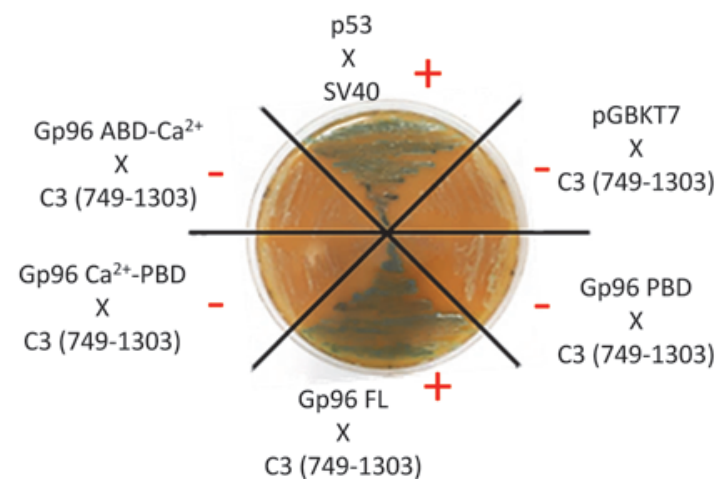

Figure 4. Mapping of Gp96 interaction with C3. (A) Linear structure of C3 ( $\beta$-chain in yellow, $\alpha$-chain in blue) and of the tested complement C3 fragments (see also Supplemental Figure 2). (B) Yeast two-hybrid assay between the C3 fragments described in $\mathbf{A}$ and full-length Gp96. A representative picture is shown. (C) Linear structure of Gp96 and the analyzed deletion mutants. ABD, ATP-binding domain; $\mathrm{Ca}^{2+}$, charged linker domain; PBD, peptide-binding domain. (D) Two-hybrid assay between complement C3 749-1,303 amino acid fragment and Gp96 deletion mutants. A representative image is shown ( $n=3$ ).

experiments with C3 KO mice, in order to better see differences in the mice groups' survival, fewer splenocytes were injected to induce the GvHD (see Methods). Altogether, these experiments in $\mathrm{C} 3 \mathrm{KO}$ animals also indicate an association between the level of complement $\mathrm{C} 3$, that of Gp96, and the GvHD severity.

\section{Discussion}

Tissue damage, resulting from the conditioning regimen before transplantation and from the alloreaction of donor $\mathrm{T}$ cells to host antigens, leads to the release of danger signals, which play a critical role in the 
A

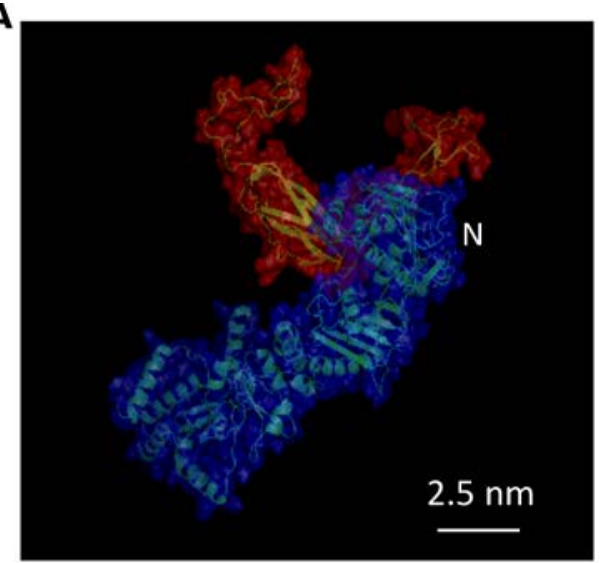

C

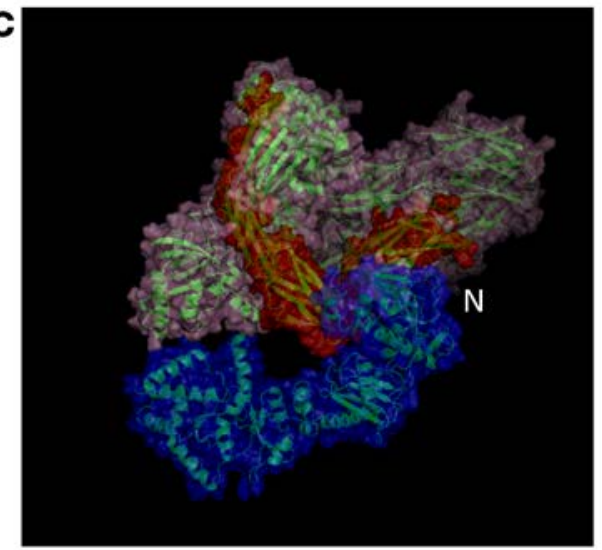

B

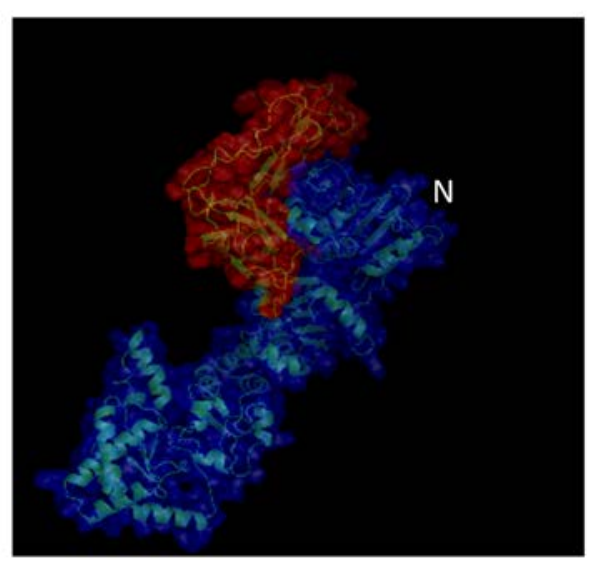

D

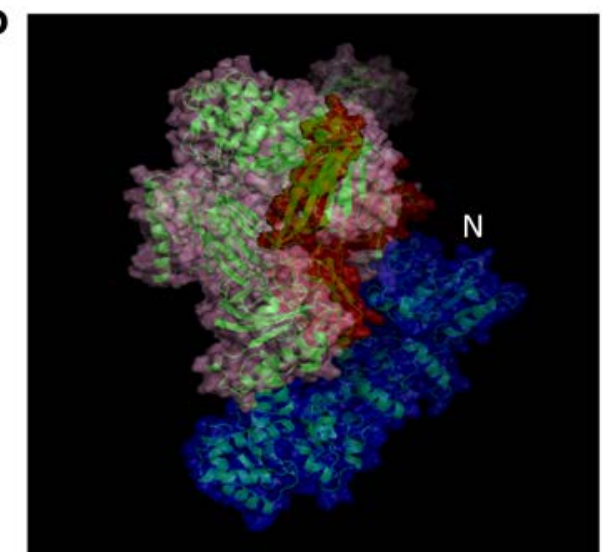

Figure 5. Atomistic models of the Gp96-C3 complex. The N-terminus of Cp96 is indicated. Each structure is represented by a transparent surface superposed to the ribbon diagram of the protein backbone. The color code is the following: red for the C3 fragment (749-955), blue for the Gp96 and pink for the C3 complementary structure (1-748; 956-1641). (A and B) Two views of the Gp96-C3 fragment model. (C and D) Two views of the Gp96 fulllength C3 model. This figure was prepared with PyMOL (http://www. pymol.org). $\mathrm{N}=\mathrm{N}$-terminal domain.

pathogenesis of $\mathrm{GvHD}$, a major complication of hematopoietic stem cell transplantation. These molecules are interesting to identify, as they represent both potential therapeutic targets and biomarkers (1). Despite the potential interest as a danger signal of Gp96, its involvement in GvHD has not yet been explored. In this work, we demonstrate in patients and mice that serum levels of Gp96 increase as GvHD develop.

This is an early and transient increase that, in the analyzed patients, did not correlate with the conditioning regimen or corticoresistance. As Hsps expression is part of a protective mechanism against adverse situations, the kinetics of $\mathrm{Gp} 96$ in the sera of patients and mice suggests that Gp96 increase is probably a consequence of intestinal damage. Indeed, there is a strong increase in Gp96 expression in duodenum biopsies of mice developing GvHD compared with controls (ref. 7 and Supplemental Figure 4).

In rheumatoid arthritis, Gp96 has been reported to be increased in the synovial fluid and to contribute to the inflammatory response (19). Also, circulating Gp96 has been reported to be increased in patients with type 1 diabetes (20), but its role in the disease pathogenesis has not been elucidated. Although different intracellular-specific partners of Gp96 have been reported (e.g., MHC class II molecules, GARP [glycoprotein A repetition predominant] that controls Treg function, or the platelet glycoprotein Ib-IX-V complex; refs. 11, 21, 22), hardly anything is known about the partners of Gp96 in the extracellular medium. In this study, we used a proteomic approach to determine that the complement C3 is a major Gp96 binding protein in the serum during GvHD. The complement represents a first line of defence against pathogens but is also now recognized as a tightly integrated surveillance and triage system playing a key role in host homeostasis (23). Cleavage of C3 is the central step in the complement activation cascade. C3b, once attached to pathogenic or apoptotic target surfaces, has major functions, notably providing a molecular platform for the formation of convertase complexes in the activation process, leading to cell lysis and favoring phagocytosis via complement receptors on phagocytes. $\mathrm{C} 3 \mathrm{~b}$ requires a tight regulation to avoid excessive activation and protect cells from complement attack under normal conditions. Under disease conditions, tissue damage can potentially trigger the complement system and in turn amplify inflammation. Our in vitro experiments with purified proteins show that Gp96 inhibits several functions of complement C3. These results are coherent with Gp96's function as a chaperone, as already reported for other Hsps, which can physically block their partners' proteolytic cleavage and/or activity (24-26).

The question remains whether, through its association with complement C3, Gp96 has a role in the physiopathology of this particular allo-immune disorder. The answer is not an easy one. First, in the serum, C3 is present at high concentrations (about $1.3 \mathrm{mg} / \mathrm{ml}$ ), whereas $\mathrm{Gp} 96$ is detected at low levels (around 

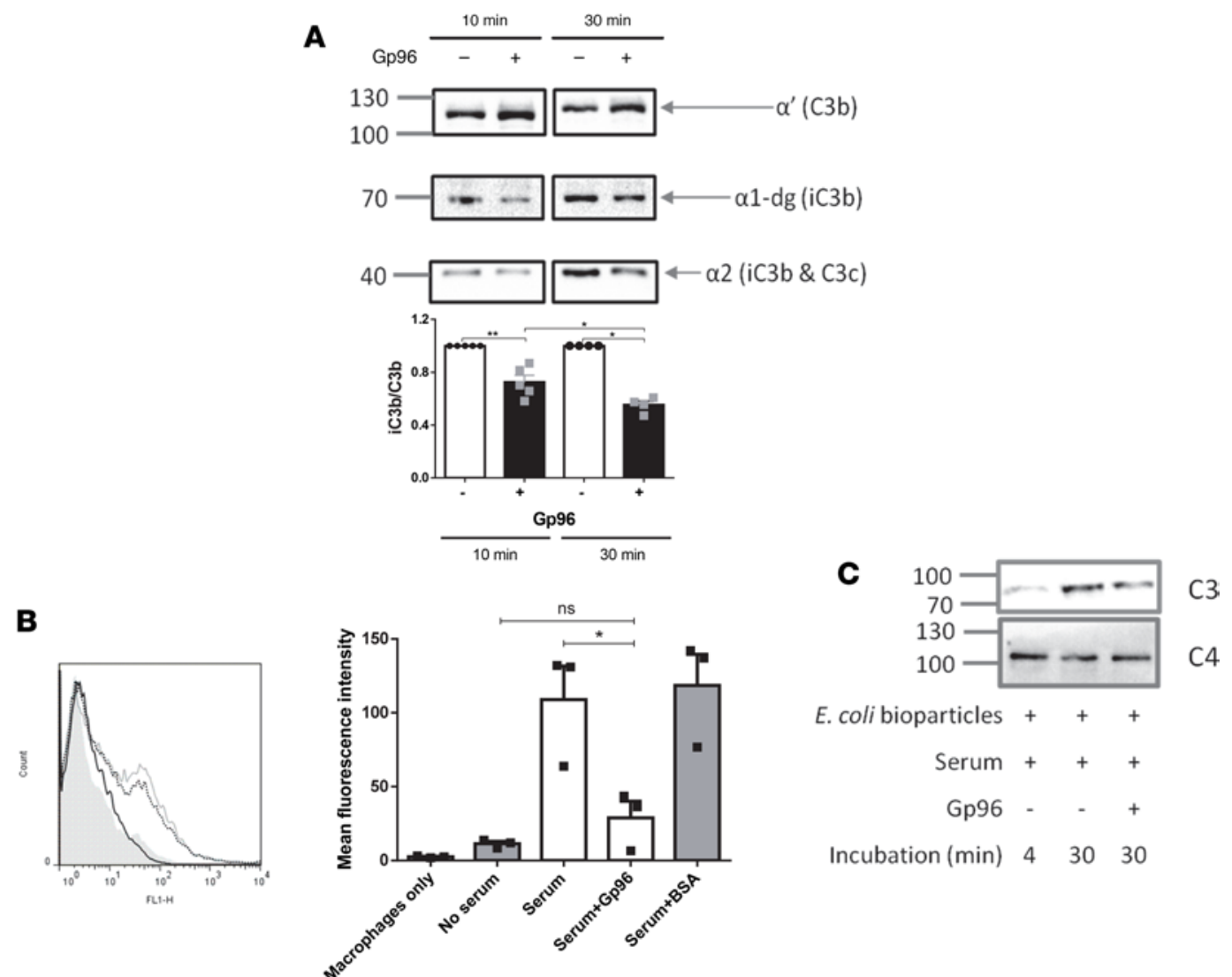

E. coli bioparticles +++

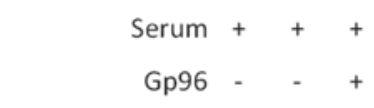

Incubation (min) $\quad 4 \quad 30 \quad 30$
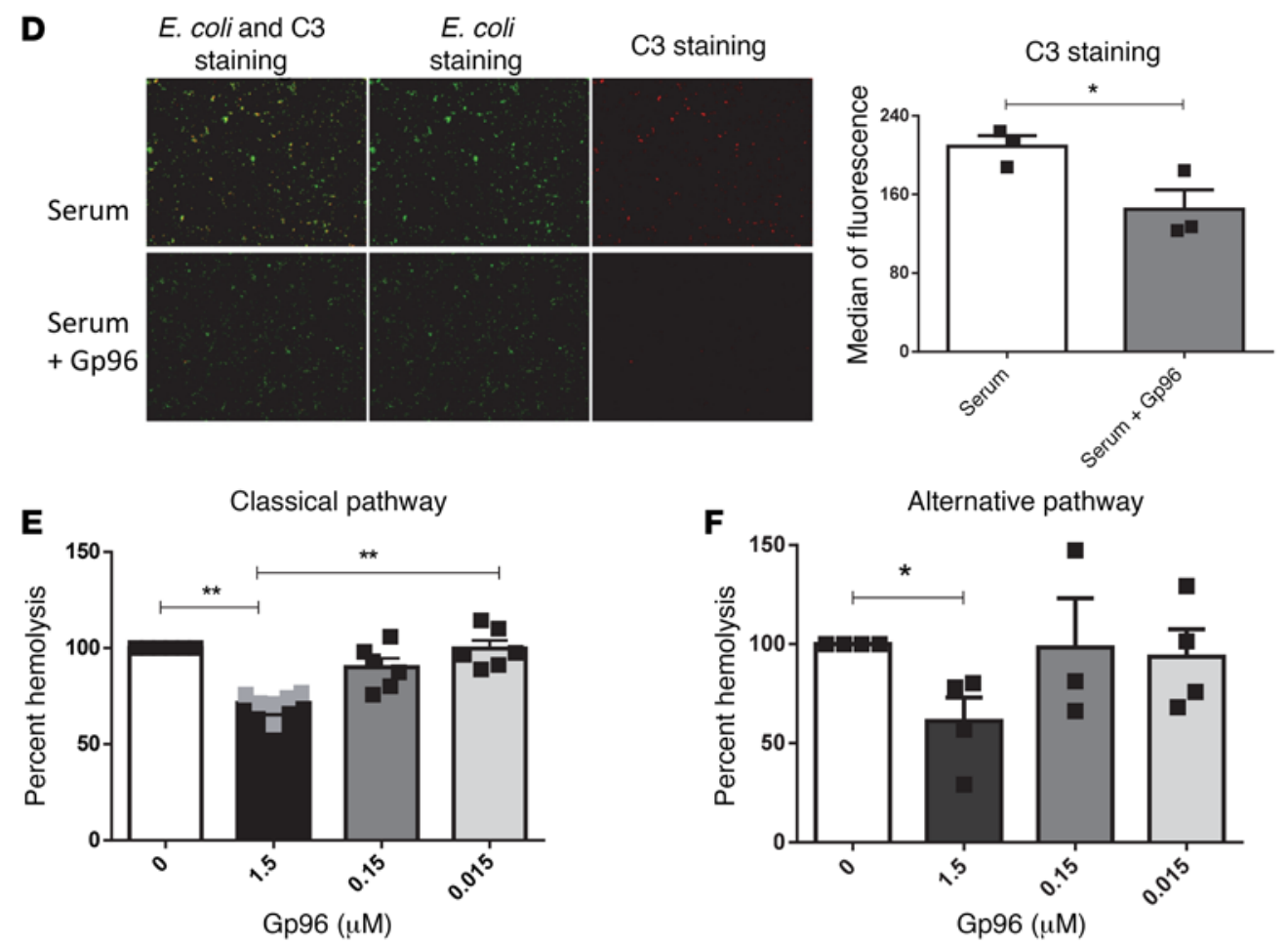

Figure 6. Gp96 effect on complement C3 activity. (A) Gp96 inhibits C3b cleavage by factors I and H. Upper panel: Western blot analysis of the $\alpha^{\prime}$-chain (C3b) and the $\alpha 2$-chain (iC3b and C3c) both labeled with anti-C3c antibody, and of the $\alpha 1$-dg-chain (iC3b) labeled with anti-C3d antibody, after C3b incubation (0.3 $\mu \mathrm{M})$ with normal human serum, supplemented with factors I and $\mathrm{H}$ with or without purified human $\mathrm{Cp} 96(1.2 \mu \mathrm{M})$. Lower panel: densitometry quantification. Cleavage without Gp96 is rationalized to 1. Statistical analysis performed using a 2-tailed Mann-Whitney $U$ test. ${ }^{*} P<0.05$; ${ }^{* *} P<0.01$ ( $n=4$ or 5). (B) Gp96 effect on opsonophagocytosis. Flow cytometry analysis of phagocytosis by human purified macrophages of Alexa Fluor 488-conjugated (AF 488-conjugated) E. coli bioparticles after opsonization by healthy human serum with or without $\mathrm{Gp} 96(1.5 \mathrm{mM})$. A representative image of AF 488 fluorescence in living cells is 
shown: the filled gray curve represents opsonization without serum, gray line with serum alone, black line with serum and Gp96, and dotted black line with serum and "BSA in buffer." Histograms of mean fluorescence intensity \pm SEM are shown. Statistical analysis performed using a one-tailed Mann-Whitney $U$ test. ${ }^{*} P<0.05(n=3)$. (C) Gp96 effect on opsonization. AF 488-E. coli bioparticles were incubated with serum with or without Gp96 (1.5 mM). C3 and C4 were determined by Western blot in supernatants after deesterification of proteins tagged on bioparticles. One representative experiment is shown ( $n=3$ ). (D) Microscopy on AF 488-bioparticles after C3 AF568 staining. Right panel: C3 bioparticle quantification (median of fluorescence of AF 568 staining \pm SEM). Statistical analysis performed using a one-tailed Mann-Whitney $U$ test. ${ }^{*} P<0.05(n=3)$. (E and F) Gp96 effect on complement activation pathways. (E) Antibody-coated sheep erythrocytes (for classical pathway) $(n=6)$ and (F) rabbit erythrocytes hemolysis (for alternative pathway) ( $n=4$ ), in presence of Gp96 or controls. Percent of hemolysis are shown. Statistical analysis performed using a 2-tailed Mann-Whitney $U$ test. ${ }^{*} P<0.05 ;{ }^{* *} P<0.01$ (E: $n=6$; $\mathbf{F}: n=4$ ).

10-50 $\mathrm{ng} / \mathrm{ml})$. One hypothesis might be that Gp96 — not only free in the serum, but also expressed in the cell membrane - can interact with complement $\mathrm{C} 3\left(\mathrm{C} 3 \mathrm{~b}\right.$ and $\left.\mathrm{C} 3\left[\mathrm{H}_{2} \mathrm{O}\right]\right)$. Indeed, our preliminary results indicate that Gp96 is abundant at the surface of epithelial intestinal cells during GvHD (Supplemental Figure 4). Second, Gp96 can affect different and apparent contradictory functions of complement C3 fragments. For instance, on the one hand, by inhibiting the C3b cleavage by the soluble regulator factor I, Gp96 can increase C3b levels. However, on the other hand, Gp96 can inhibit major functions of C3b such as opsonization and formation of the convertase complexes. Interestingly, a similar incongruent impact on $\mathrm{C} 3 \mathrm{~b}$ has been reported for the Staphylococcal complement inhibitor (SCIN) family of proteins, which allow the bacteria to escape the complement attack. Remarkably, the SCIN proteins bind to the same region of complement C3 identified here for Gp96 interaction (27). This region that includes the $\alpha$ 'NT, MG6, and MG7 domains (Supplemental Figure 2) has been proposed as a functional C3 hot spot, important for binding several regulators (27) and, notably, factor H. For $S$. aureus, the final consequence, despite the paradoxical effect on $\mathrm{C} 3 \mathrm{~b}$ inactivation, is complement inhibition. Thus, in a similar manner, during GvHD, Gp96 could inhibit C3b and regulate complement C3 to avoid excessive damage and inflammation - the negative counterpart of this inhibition being a lower effect of complement on pathogens.

Mouse models of GvHD have shown that C3 is an important mediator of GvHD pathogenesis, suggesting again that Gp96, by regulating C3, may have a beneficial effect against GvHD development. C3-deficient recipient mice develop a less severe disease (18). At the opposite, when C3-deficient mice are used as donors, we found that the receiving mice developed a more severe GvHD and had increased levels of $\mathrm{C} 3$ - and Gp96 - in their serum. We have no explanation at present for these results. Independently of this unresolved issue, our results confirmed the link between C3 and Gp96 serum levels and the severity of the disease. Interestingly, our data using a peptide aptamer inhibitor of Gp96 indicates a strong aggravation of GvHD development (data not shown). Unfortunately, these results are difficult to interpret, as there was also an increase in animal death in the syngeneic group. This strongly suggests that this toxicity obtained with the Gp96 inhibitor is not just the consequence of the inhibition of Gp96's effect on complement C3 but probably the result of all intra- and extracellular functions described for this essential chaperone whose $\mathrm{KO}$ is lethal for the animal (28).

In conclusion, our results indicate that Gp96 can chaperone complement C3 in the serum during GvHD development and that Gp96 serum levels deserve to be clinically tested as a marker of GvHD severity. A clinical study has started where the levels of Gp96 and complement C3 are being determined both in serum and in intestinal biopsies before and after bone marrow transplantation.

\section{Methods}

Study design. Serum Gp96 was quantified by ELISA in patients developing a GvHD at different times after hematopoietic stem cell transplantation. All patients provided an informed consent for biological research purposes. The increase in Gp96 levels was confirmed by Western blot in a mouse model of GvHD $(\mathrm{C} 57 \mathrm{BL} / 6 \mathrm{BALB} / \mathrm{c})$. The proteins associated to Gp96 in the serum of mice during GvHD development were identified after immunoprecipitation of Gp96 and analysis of SDS-PAGE bands by MS.

The interaction between Gp96 and the complement C3 and/or its cleaved fragment C3b was further characterized by biolayer interferometry, yeast 2 hybrid assay and in silico modeling. The impact of the Gp96-C3 interaction on the cleavage of C3 by factor I was analyzed by Western blot. We also analyzed Gp96 impact on C3 functions (i.e., inhibition of opsonophagocytosis and of activation by the alternative and the classical pathway).

To confirm in vivo that Gp96 may be a chaperone of complement C3 associated with GvHD severity, complement $\mathrm{C} 3 \mathrm{KO}$ animals were used to induce GvHD, either as recipient mice or as donor mice (Gp96 $\mathrm{KO}$ animals are not viable). 
A
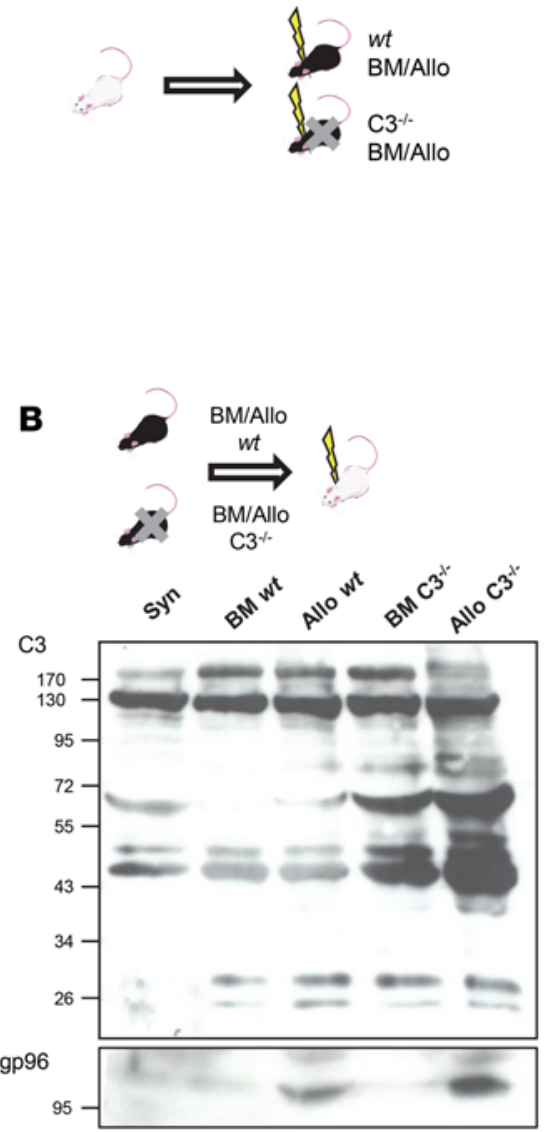

C

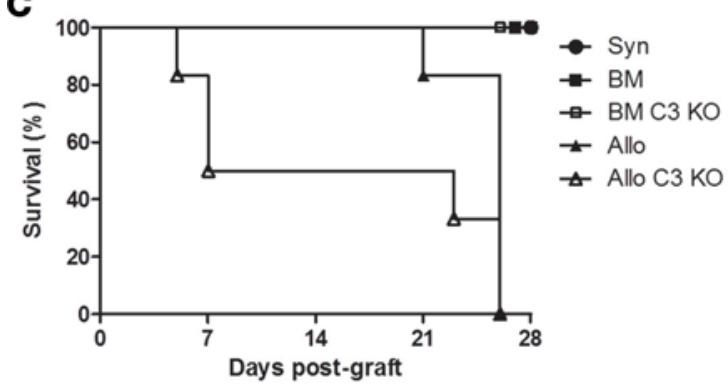

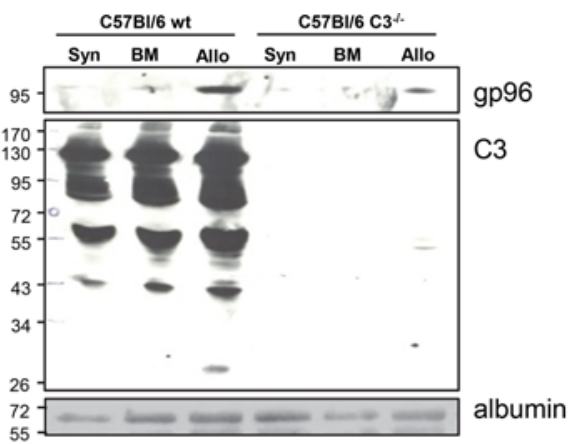
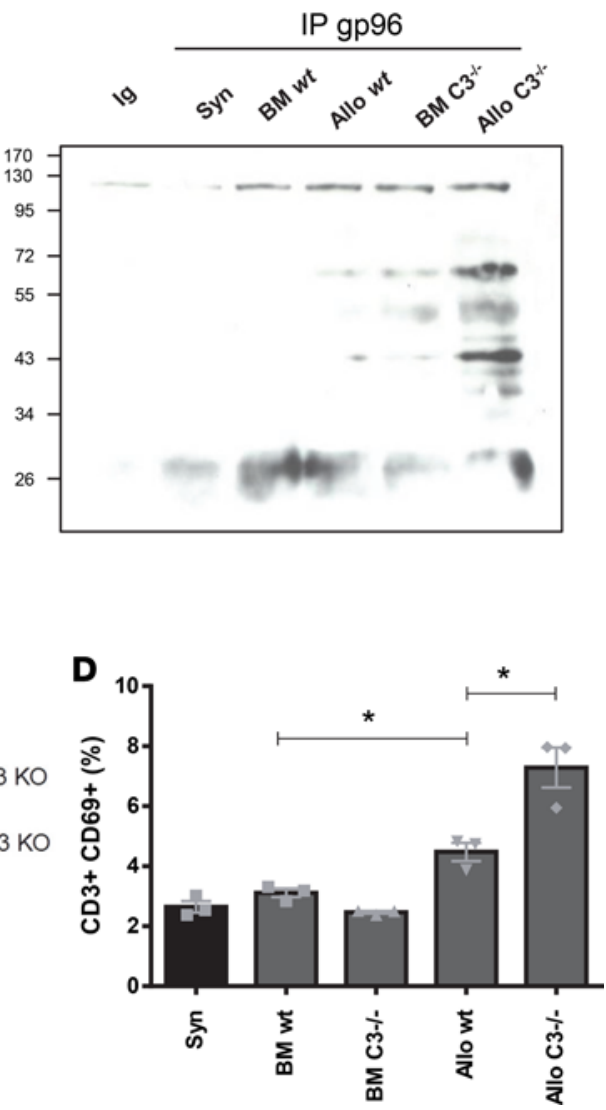

Figure 7. Study of Gp96 in GvHD using complement C3 KO animals. (A) Left, schematic representation of the model of GvHD used with a $\mathrm{C3}^{-/-}$recipient: $\mathrm{FvB} / \mathrm{N} \rightarrow \mathrm{WT}$ or $\mathrm{C3}^{-/-} \mathrm{C} 57 \mathrm{BL} / 6$. Right, Western blot analysis of $\mathrm{C} 3$ and $\mathrm{Cp} 96$ in day 7 sera of WT or $\mathrm{C3}^{-1-}$ mice, developing GVHD (Allo) or not (Syn and BM). (B) Top, schematic representation of the mice model with a $\mathrm{C3}^{-/-}$donor: WT or $\mathrm{C3}^{-/-} \mathrm{C} 57 \mathrm{BL} / 6 \rightarrow \mathrm{BALB} / \mathrm{c}$. Lower left panel, Western blot analysis of $\mathrm{C3}$ and $\mathrm{Gp96}$ in day 7 sera of mice receiving a WT or a $\mathrm{C}^{-/-}$graft, developing CVHD (Allo) or not (Syn and BM). Samples from 7 animals per group were pooled. Lower right panel, immunoprecipitation of Gp96 in the sera from the different described animals groups was followed by complement C3 immunoblotting. One representative experiment out of 3 performed is shown. (C) Survival of the mice belonging to the different groups described in $\mathbf{B},(n=4)$ from 2 independent experiments. (D) Percentage of activated, splenic T cells $\left(\mathrm{CD}^{+} \mathrm{CD69}^{+}\right) 7$ days after hematopoietic stem cell transplantation in mice receiving either a WT or a $\mathrm{C3}^{-/-}$graft, developing GVHD (Allo) or not (Syn and BM). Statistical analysis performed using a one-tailed Mann-Whitney $U$ test. ${ }^{*} P<0.05(n=3)$.

The animals were always randomly assigned to each group. For ethical reasons, the animals were sacrificed when the clinical score was $>6$.

Mice and acute GvHD models. BALB/c $\left(\mathrm{H}-2^{\mathrm{d}}\right), \quad \mathrm{FvB} / \mathrm{N} \quad\left(\mathrm{H}-2^{\mathrm{q}}\right)$, $\mathrm{C} 57 \mathrm{BL} / 6 \mathrm{WT}$ and $\mathrm{C}^{-1-}\left(\mathrm{H}-2^{\mathrm{b}}\right)$ female mice, between 7 and 10 weeks old, were purchased from Charles River Laboratories. The day before transplantation, recipients were lethally irradiated (9 Gy).

Grafts were prepared from bone marrow (for hematopoietic stem cells) and spleen (for mature T lymphocytes) of donor animals. Cells were injected retro-orbitally into irradiated recipients. Mice received either a syngeneic graft (Syn, $1 \times 10^{6}$ bone marrow cells and $1 \times 10^{7}$ splenocytes from haplotype-identical mice), a bone marrow allogeneic graft (BM, $1 \times 10^{6}$ bone marrow cells from haplotype-different mice), or a bone marrow + spleen allogeneic graft (Allo, $1 \times 10^{6}$ bone marrow cells and $1 \times 10^{7}$ splenocytes from haplotypedifferent mice). For experiments with $\mathrm{C}^{-1-}$ mice, $2 \times 10^{6}$ splenocytes were injected in order to better see differences in survival. The ethic committee of the Université de Bourgogne approved all animal protocols.

Gp96 ELISA in GvHD patient sera. A total of 46 patients who underwent an allogeneic hematopoietic stem cell transplantation with bone marrow or peripheral stem cell grafts following myeloablative conditioning in Saint-Antoine and Necker Hospitals and the Institute Gustave Roussy, Paris, entered the study. All patients provided an informed consent for biological research purposes. The study was conducted according to the procedures of the Declaration of Helsinki and the local ethic committee rules. Diagnosis and grading of acute GvHD was performed as described (29). 
Sera from patients was analyzed using HSP90b1 ELISA kit (Euromedex).

Flow cytometry. Spleens were removed at D7 after graft, and splenocytes were stained with anti-mouse F4/80 (catalog BLE123122; Biolegend) and anti-Gp96 (catalog ADI-SPA-850-488; Enzo Life Sciences) or anti-CD3 (catalog 16-0037-81; Biolegend) and anti-CD69 (catalog 17-0691-82; eBioscience) antibodies. Acquisition was performed on LSR II cytometer (BD Biosciences). For phagocytosis experiment (see below), macrophages were gated on FCS/SSC, and fluorescence was detected at $488 \mathrm{~nm}$.

Histological study. Small intestine sections were taken at D7 after graft, fixed with $4 \%$ paraformaldehyde overnight, and included in paraffin. For immunohistochemical analysis, sections were deparaffinized and stained for Gp96 expression using a polyclonal anti-Gp96 antibody (catalog ADI-SPA-851; Enzo Life Sciences). Secondary anti-rabbit HRP antibody (catalog 669630; Jackson Immunoresearch) was added, followed by development with NovaRED Peroxidase Substrate Kit (Vector laboratories).

Western blotting, immunoprecipitation, and proteomics. Expression of Gp96 and complement C3 in mice's sera was evaluated using polyclonal anti-Gp96 (catalog ADI-SPA-851; Enzo Life Sciences) and anti-C3 (catalog HP8012; HyCult Biotech). In some experiments, recombinant Gp96 produced in the human cell line HEK293 (BioVendor) and human recombinant HSP90 (Enzo Life Sciences) were used. Fragments of C3 were identified using anti-C3d and anti-C3c antibody (catalogs A0063 and A0062; Dako). Secondary anti-rabbit HRP antibody was used (catalog 669630; Jackson Immunoresearch).

For proteomics analysis, serum samples were precleared with protein A- and G-coupled agarose beads (Millipore). Equal contents in proteins were incubated with Exactacruz B beads (Santa Cruz Biotechnology Inc.) previously coated with anti-Gp96 antibody (clone 9G10; catalog ADI-SPA-850; Enzo Life Sciences). Anti-GFP antibody (clone FL; catalog sc8334; Santa Cruz Biotechnology Inc.) was used as a nonrelevant immunoglobulin.

Nonspecific staining of electrophorated proteins was done using a Coomassie blue solution (Sigma). Bands of interest were sampled. Proteins were digested with trypsin and peptides extracted and analyzed by MS/MS (30).

Biolayer interferometry. Proteins were biotinylated as described (24). The biotinylated protein was immobilized onto streptavidin biosensor tips and dipped into wells containing the buffer with the analyte of interest. Sensorgrams were background corrected, smoothed with the Savitzky-Golay algorithm, and analyzed using OctetRED instrument software (ForteBio Data Analysis version 7.1.).

Yeast two-hybrid assay. Vectors pGBKT7 and pGADT7 were used (Clontech). Complement C3 and Gp96 coding plasmids were purchased from the Mammalian Genome Collection (LGC Standards). Corresponding coding sequence of following fragments of C3 were cloned: C3 (25-666), C3 (749-1,663), C3 (749-1,303), C3 (1,321-1,663), C3 (749-954) and C3 (955-1,303) into NdeI/NcoI sites of pGADT7. Coding sequence of Gp96 full-length and deletion mutants were cloned into NdeI/BamHI sites of pGBKT7. pGADT7-SV40, which codes for the GAL4 activation domain fused with SV40 large T antigen, was used as a positive control; empty pGADT7 was used as a negative control. All pGADT7- and pGBKT7-derived vectors were transformed into Y187 and Y2HGold yeast strains using Clontech Yeastmaker (Clontech).

In silico modeling. The structure of Gp96 (PDB code: 2O1U chain A) (31) was first docked with the isolated C3 fragment (amino acids 749-955) (PDB code: 2A73) (32) using the package RosettaDock (https://www.rosettacommons.org). We performed 100,000 runs of low-resolution docking with random rotations of Gp96. The structures found were ranked using the RosettaDock scoring function. The best structure computed from the high-resolution docking was optimized in vacuum and further relaxed by molecular dynamics (MD) simulations using Gromacs (33).

Classical and alternative complement pathways. Healthy volunteer sera were incubated at $37^{\circ} \mathrm{C}$ for 1 hour with Gp96 produced in the human cell line HEK293 (BioVendor). For the classical pathway, the mixture was added to standardized sheep erythrocytes coated with antibodies (Delta Biologicals) for 1 hour at $37^{\circ} \mathrm{C}$. For the alternative pathway, the mixture was added to $30 \times 10^{6}$ rabbit erythrocytes in gelatin veronal buffer (without calcium and magnesium) containing $13 \mathrm{mM}$ of $\mathrm{Mg} /$ EGTA to inhibit the classical pathway (all from CompTech) for 30 minutes at $37^{\circ} \mathrm{C}$. The absorbance of the supernatants was read at $405 \mathrm{~nm}$.

Opsonophagocytosis. Peripheral blood mononuclear cells were separated using Ficoll and harvested in X-VIVO 15 medium (Ozyme) with macrophage CSF (Miltenyi Biotec). For opsonization, Alexa Fluor (AF) 488-conjugated E. coli bioparticles (Invitrogen) were used. After 1 hour, macrophages were washed in PBS, extracellular fluorochromes were quenched by trypan blue, and fluorescence was analyzed. For the release of tagged C3 bioparticles, we used a previously reported method (34). 
Statistics. Data are expressed as mean \pm SEM. Nonparametric ANOVA and 1- or 2-tailed Mann-Whitney $U$ tests were used for the statistical analyses according to the data context. Data were analyzed using Graphpad Prism (Graphpad Prism 6 software).

Study approval. The ethics committee of the Burgundy University approved all animal protocols. The study in patients was conducted according to the procedures of the Declaration of Helsinki and the local consultative ethics committee rules. All patients provided an informed consent for biological research purposes.

\section{Author contributions}

AS, ALJ, and KC performed most experiments and analyzed the data. A. Hazoumé performed the yeast two-hybrid assay. MS and CB performed Western blots. GM and RS did the biolayer Interferometry experiments. A. Hammann and GJ were in charge of the FACS analysis. P. Ducoroy performed proteomic experiments. P. Delarue and PS realized in silico modeling studies. ES helped writing the paper and designing experiments. CCL, MAD, MTR, and $\mathrm{OH}$ collected human samples and helped discussing the paper. EK and CG supervised the overall project, analyzed the data, and wrote the manuscript.

\section{Acknowledgments}

We thank Galit Rotman and Anat Oren (Compugen Ltd., Tel Aviv, Israel) for giving us the CGEN-25007 peptide aptamer and for their advise. We thank P. Saas (INSERM U1098, Besançon, France), E. Robinet (INSERM U748, Strasbourg, France), J. Haiech (Université de Strasbourg), and E. Schmitt (INSERM U866, Dijon, France) for helpful discussions. This work was supported by grants from the Institut National du Cancer, Agence Nationale de la Recherche, Ligue Nationale Contre le Cancer ("Labeled teams" to CG and $\mathrm{OH}$ ), the Association pour la Recherche sur le Cancer (ARC-labeled team to CG), and the Conseil Régional de Bourgogne. The work was also supported by a French Government grant managed by the French National Research Agency under the program "Investissements d'Avenir" with reference ANR11-LABX-0021 (LabEX LipSTIC). We thank the European Union programme FEDER for their financial support. ALJ has a fellowship from La Ligue Nationale contre le Cancer and GM from l'ARC.

Address correspondence to: Carmen Garrido, INSERM U866, UFR des Sciences de Santé, 7 boulevard Jeanne d'Arc, 21079 Dijon, France. Phone: 33.3.80.39.32.84; E-mail: cgarrido@u-bourgogne.fr.

1. Ramadan A, Paczesny S. Various forms of tissue damage and danger signals following hematopoietic stem-cell transplantation. Front Immunol. 2015;6:14.

2. Wingard JR, et al. Long-term survival and late deaths after allogeneic hematopoietic cell transplantation. J Clin Oncol. 2011;29(16):2230-2239.

3. Ferrara JL, Levine JE, Reddy P, Holler E. Graft-versus-host disease. Lancet. 2009;373(9674):1550-1561.

4. Ferrara JL, et al. Regenerating islet-derived 3-alpha is a biomarker of gastrointestinal graft-versus-host disease. Blood. 2011;118(25):6702-6708.

5. Garrido C, Solary E. A role of HSPs in apoptosis through "protein triage"? Cell Death Differ. 2003;10(6):619-620.

6. Jarvis M, Marzolini M, Wang XN, Jackson G, Sviland L, Dickinson AM. Heat shock protein 70: correlation of expression with degree of graft-versus-host response and clinical graft-versus-host disease. Transplantation. 2003;76(5):849-853.

7. Joly AL, et al. The HSP90 inhibitor, 17AAG, protects the intestinal stem cell niche and inhibits graft versus host disease development. Oncogene. 2016;35(22):2842-2851.

8. Stuehler C, et al. Selective depletion of alloreactive T cells by targeted therapy of heat shock protein 90: a novel strategy for control of graft-versus-host disease. Blood. 2009;114(13):2829-2836.

9. Tsan MF, Gao B. Heat shock proteins and immune system. J Leukoc Biol. 2009;85(6):905-910.

10. Yang Y, Li Z. Roles of heat shock protein gp96 in the ER quality control: redundant or unique function? Mol Cells. $2005 ; 20(2): 173-182$

11. Zhang Y, et al. GP96 is a GARP chaperone and controls regulatory T cell functions. J Clin Invest. 2015;125(2):859-869.

12. Ochayon DE, Mizrahi M, Shahaf G, Baranovski BM, Lewis EC. Human $\alpha 1$-Antitrypsin Binds to Heat-Shock Protein gp96 and Protects from Endogenous gp96-Mediated Injury In vivo. Front Immunol. 2013;4:320.

13. Pawaria S, Binder RJ. CD91-dependent programming of T-helper cell responses following heat shock protein immunization. Nat Commun. 2011;2:521.

14. Singh-Jasuja H, et al. The heat shock protein gp96 induces maturation of dendritic cells and down-regulation of its receptor. Eur J Immunol. 2000;30(8):2211-2215.

15. Doody AD, Kovalchin JT, Mihalyo MA, Hagymasi AT, Drake CG, Adler AJ. Glycoprotein 96 can chaperone both MHC class I- and class II-restricted epitopes for in vivo presentation, but selectively primes CD8+ T cell effector function. J Immunol. 2004;172(10):6087-6092.

16. Suto R, Srivastava PK. A mechanism for the specific immunogenicity of heat shock protein-chaperoned peptides. Science. 
1995;269(5230):1585-1588.

17. Ricklin D, Lambris JD. Therapeutic control of complement activation at the level of the central component C3. Immunobiology. 2016;221(6):740-746.

18. Ma Q, et al. Reduced graft-versus-host disease in C3-deficient mice is associated with decreased donor Th1/Th17 differentiation. Biol Blood Marrow Transplant. 2012;18(8):1174-1181.

19. Huang QQ, et al. Glycoprotein 96 perpetuates the persistent inflammation of rheumatoid arthritis. Arthritis Rheum. 2012;64(11):3638-3648.

20. Pagetta A, Folda A, Brunati AM, Finotti P. Identification and purification from the plasma of Type 1 diabetic subjects of a proteolytically active Grp94Evidence that Grp94 is entirely responsible for plasma proteolytic activity. Diabetologia. 2003;46(7):996-1006.

21. Schaiff WT, Hruska KA, McCourt DW, Green M, Schwartz BD. HLA-DR associates with specific stress proteins and is retained in the endoplasmic reticulum in invariant chain negative cells. J Exp Med. 1992;176(3):657-666.

22. Staron M, et al. Heat-shock protein gp96/grp94 is an essential chaperone for the platelet glycoprotein Ib-IX-V complex. Blood. 2011;117(26):7136-7144.

23. Ricklin D. Manipulating the mediator: modulation of the alternative complement pathway C3 convertase in health, disease and therapy. Immunobiology. 2012;217(11):1057-1066.

24. Arlet JB, et al. HSP70 sequestration by free $\alpha$-globin promotes ineffective erythropoiesis in $\beta$-thalassaemia. Nature. 2014;514(7521):242-246.

25. de Thonel A, et al. HSP27 controls GATA-1 protein level during erythroid cell differentiation. Blood. 2010;116(1):85-96

26. Ribeil JA, et al. Hsp70 regulates erythropoiesis by preventing caspase-3-mediated cleavage of GATA-1. Nature. 2007;445(7123):102-105.

27. Ricklin D, et al. A molecular insight into complement evasion by the staphylococcal complement inhibitor protein family. J Immunol. 2009;183(4):2565-2574

28. Wanderling S, et al. GRP94 is essential for mesoderm induction and muscle development because it regulates insulin-like growth factor secretion. Mol Biol Cell. 2007;18(10):3764-3775.

29. Przepiorka D, et al. Prevention of graft-versus-host disease with anti-CD5 ricin A chain immunotoxin after CD3-depleted HLAnonidentical marrow transplantation in pediatric leukemia patients. Bone Marrow Transplant. 1995;16(6):737-741.

30. Pflieger D, et al. Comparative proteomic analysis of extracellular matrix proteins secreted by two types of skin fibroblasts. Proteomics. 2006;6(21):5868-5879.

31. Dollins DE, Warren JJ, Immormino RM, Gewirth DT. Structures of GRP94-nucleotide complexes reveal mechanistic differences between the hsp90 chaperones. Mol Cell. 2007;28(1):41-56.

32. Janssen BJ, et al. Structures of complement component C3 provide insights into the function and evolution of immunity. Nature 2005;437(7058):505-511.

33. Hess B, Kutzner C, van der Spoel D, Lindahl E. GROMACS 4: Algorithms for Highly Efficient, Load-Balanced, and Scalable Molecular Simulation. J Chem Theory Comput. 2008;4(3):435-447.

34. Gordon DL, Rice J, Finlay-Jones JJ, McDonald PJ, Hostetter MK. Analysis of C3 deposition and degradation on bacterial surfaces after opsonization. J Infect Dis. 1988;157(4):697-704. 